\title{
Relationship between Haemoglobin A1c and Short Term Outcome in Patients with ST-elevation Myocardial Infraction (STEMI)
}

\author{
S.M. Kamrul Hasan ${ }^{1}$, Md. Rezaul Karim ${ }^{2}$, Md. Anisur Rahman Khan ${ }^{3}$, Tareq Ahmed Chowdhury ${ }^{1}$, Khondokar \\ Asaduzzam $^{3}$, Md. Salahuddin Ulabbi ${ }^{1}$, Nadira Sultana Kakoly ${ }^{4}$
}

\begin{abstract}
:
Introduction: Every year, 17.1 million lives are claimed by the global burden of cardiovascular disease (CVD), $82 \%$ of which are in the developing world. Glycated hemoglobin (HbA1c), even at levels considered in the "normal" range, emerged as an independently significant predictor of heart-disease events, stroke, and death over more than a decade.

Methodology: After applying the inclusion and exclusion criteria, 113 patients with ST segment elevation myocardial infarction were enrolled in this study after taking informed written consent from the patient or attending guardian. Follow up was done during hospital stay for mortality, arrhythmia, cardiogenic shock, cardiac arrest, congestive heart failure, mechanical complication (eg ventricular septum rupture, wall rupture), left ventricular systolic dysfunction, stroke etc.
\end{abstract}

Result: The median age of patients was 53.4 years (range 22 to 85 years), patients with an $\mathrm{HbA1c}>6.5 \%$ were slightly older than those with $\mathrm{HbA1c}<6.5 \%(53.1$ vs. 54.6$)$, and $82 \%$ were male; and $43 \%$ had an $\mathrm{HbA} 1 \mathrm{c}>6.5 \%$. Patients with elevated HbA1c had more LVSD $(54 \%)(p=0.022)$, heart failure $(81 \%)(p=<.001)$.

However, patients with HbA1c $>6.5 \%$ were more likely to have cardiogenic shock as an outcome but it is not statistically significant ( $p=.528)$, whereas cardiac arrest, arrythmia and mechanical complication were more among HbA1c <6.5 group but it was not statistically significant.

During hospital stay 16 (14\%) patients died. Mortality was much higher among all STEMI patients, those with elevated hemoglobin A1c level as an outcome compared to patients with normal haemoglobin A1c level (26\% vs. $5 \%)(p=.002)$.

Conclusion : STEMI patients who has haemoglobin HbA1c level $<6.5$ have better in hospital outcome compared to elevated (>6.5) haemoglobin A1c level.

(Bangladesh Heart Journal 2015; 30(1) : 29-32)

Introduction :

Coronary artery disease (CAD) is the leading cause of mortality and morbidity in western society and is a worldwide epidemic. In 2001, worldwide death due to ischemic heart disease (IHD) was $11.8 \%$ in low income countries and $17.3 \%$ in high income countries ${ }^{1}$.

Every year, 17.1 million lives are claimed by the global burden of cardiovascular disease (CVD) - 82\% of which are in the developing world. Myocardial infarction is one of the leading cause of death in Bangladesh mostly in

1. National Institute of Cardiovascular Diseases, Dhaka. E-mail: hasandrkamrul@gmail.com

2. Upazilla Health Complex, Dhamrai, Dhaka.

3. Sir Salimullah Medical College. Dhaka.

4. James. P. Grant School of Public health, BRAC University, Dhaka the $4^{\text {th }}$ decade of life. In a study of 2690 patients, in hospital mortality was $11.8 \%$. The main cause of death was pump failure and ventricular fibrillation ${ }^{2}$.

Considering the $\mathrm{HbA1c}$ level as a risk factor, it was found that there was significant relationship between $\mathrm{HbA1C}$ level and in hospital mortality among STEMI $(P=0.006){ }^{3}$

Recent evidence has shown that chronic glucose dysregulation, assessed by HbA1c levels, is also of prognostic value with regard to future cardiovascular disease and congestive heart failure ${ }^{4}$. Glycated hemoglobin $(\mathrm{HbA} 1 \mathrm{c})$, even at levels considered in the "normal" range, emerged as an independently significant predictor of heart-disease events, stroke, and death over more than a decade. ${ }^{5}$ 
Methodology : All patients admitted in cardiology department with STEMI were evaluated by history taking, standardised clinical examination, Electrocardiogram, Troponin-I, Echocardiogram, blood tests for urea and electrolytes and full blood count.and other necessary investigations as required. On the basis of inclusion and exclusion criteria, patients were enrolled in this study.

Follow up was done for any complication like development of cardiogenic shock, cardiac arrest, appearance of any new murmur, symptoms/signs of heart failure (eg dyspnea, orthopnea, basal crepitation ), monitoring of ECG for any arrythmia (eg atrial fibrilation, atrial flutter, ventricular tachycardia, ventricular fibrilation, premature ventricular contraction, etc)

All the patients at first underwent $2 \mathrm{D}$ and $\mathrm{M}$ mode echocardiography and analysed for chamber dimenssion, ventricular wall thickness, wall motion abnormalities and systolic function. Color flow and Doppler imaging also done to see the mechanical complication, wall motion abnormality were graded from normal to dyskinesia. Ejection fraction was calculated automatically from the above indices. Ejection fraction were taken in two views, parasternal long axis and short axis at the level of tip of the mitral valve leaflets and papilary muscle level and then final EF were taken from their average to reduce the measurement error. Left ventricular systolic impairment was assessed subjectively in all patients on a scale of normal/mild/moderate/severe systolic impairment. Left ventricular systolic dysfunction (LVSD) was considered to be present when left ventricular ejection fraction was $<55 \%$ or left ventricular systolic impairment was graded as mild. ${ }^{6}$

A non-fasting blood sample was taken within 24 hours of admission for the measurement of random blood glucose and HbA1c level. All routine blood samples were analyzed on the day of sample collection as per standard clinical practice.

HbA1c was measured using high-performance liquid chromatography and $\mathrm{HbA} 1 \mathrm{c}$ of $4-6.5 \%$ was considered as normal. HbA1c level d" 6.5 was diagnosed as normal and above this value was leveled as elevated HbA1c. ${ }^{7}$

Analysis was done by using SPSS version 11.5 software program. Confidence interval was set at 95\% level. Result were considered to be statistically significant if $p$-value was $<0.05$. In the statistical analysis, the demographic characteristics age and sex were expressed as proportions. Pearson Chi-square test was used to compare between the categories between elevated and normal glycated haemoglobin A1c, with the interest of outcome.
Result : Among elevated HbA1c group patients, 35 (71\%) were male and $14(29 \%)$ were female. Among normal HbA1c group patients, 58 (91\%) were male and 6(9\%) were female. Mean age was 53.75+/-11.

$\square$ Haemoglobin level $\leq 6.5$

$\square$ Haemoglobin level $>6.5$

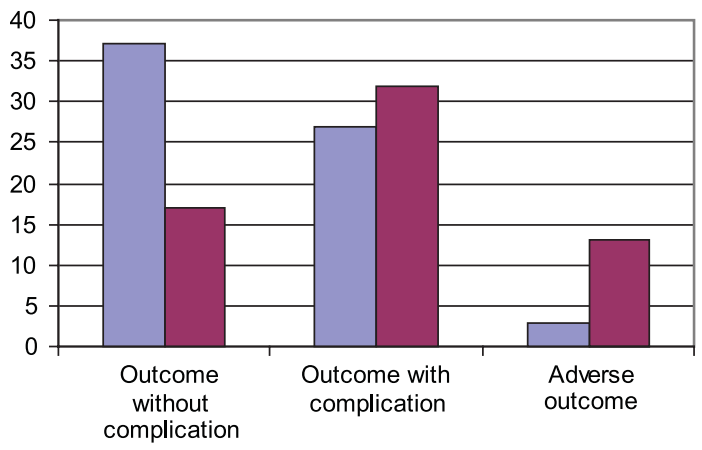

Fig- 1 shows that patients who has haemoglobin $\mathrm{HbAc}$ level $\leq 6.5$ have better in hospital outcome compared to elevated (>6.5) haemoglobin A1c level.

Table- I

In hospital outcome among normal and elevated HbA1c level

\begin{tabular}{|c|c|c|c|}
\hline \multirow{2}{*}{$\begin{array}{l}\text { Outcome of } \\
\text { interest }\end{array}$} & \multicolumn{2}{|c|}{ HbA1c level } & \multirow[t]{2}{*}{ Total $\mathrm{N}=113$} \\
\hline & $\begin{array}{c}\leq 6.5(n=64) \\
n(\%)\end{array}$ & $\begin{array}{c}>6.5(n=49) \\
n(\%)\end{array}$ & \\
\hline Cardiac arrest & $2(100 \%)$ & $0(0 \%)$ & 2 \\
\hline Cardiogenic shock & $5(45 \%)$ & $6(55 \%)$ & 11 \\
\hline Arrythmia & $11(58 \%)$ & $8(42 \%)$ & 19 \\
\hline Heart failure & $5(19 \%)$ & $21(81 \%)$ & 26 \\
\hline Mechanical & $2(100 \%)$ & $0(0 \%)$ & 2 \\
\hline $\begin{array}{l}\text { LV systolic } \\
\text { dysfunction }\end{array}$ & $27(46 \%)$ & $32(54 \%)$ & 59 \\
\hline
\end{tabular}

Table- I shows the distribution of in hospital outcome among the study subjects when compared the patients with normal $\mathrm{HbA} 1 \mathrm{c}$ and elevated HbA1c. Patients with elevated $\mathrm{HbA} 1 \mathrm{c}$ are more frequently had cardiogenic shock, heart failure and LV systolic dysfunction (55\%, $81 \%$ and 54\% respectively) and cardiac arrest, arrythmia and mechanical complication are more common among patients with normal hemoglobin A1c level. 
Table- II

In hospital mortality as an outcome of the study subjects according to HbAlc level

\begin{tabular}{lccc}
\hline HbA1c level & In hospital mortality as an outcome & P-value \\
absentn(\%) & $\begin{array}{c}\text { In hospital mortality } \\
\text { presentn(\%) }\end{array}$ & Total N=113 & 0.002 \\
\hline d"6.5 & $61(95 \%)$ & $3(5 \%)$ & 64 \\
$>6.5$ & $36(74 \%)$ & $13(26 \%)$ & 49 \\
\hline Total & 97 & 16 & 113 \\
\hline
\end{tabular}

$\mathrm{P}$ value reached from Pearson Chi-square test

Table- II shows that among all STEMI patients, those with elevated hemoglobin A1c level were more likely to have death as an outcome compared to patients with normal haemoglobin A1c level (26\% vs. $5 \%) .(p=.002)$.

\section{Discussion :}

This study evaluated the prognostic value of $\mathrm{HbA} 1 \mathrm{c}$ in patients with STEMI. Elevated $\mathrm{HbA1c}$ was associated with a worse prognosis. This prognostic cohort study shows that $\mathrm{HbA} 1 \mathrm{c}$ levels $>6.5 \%$ was associated with in hospital outcome in STEMI patients. This association was significant among patients who has heart failure and left ventricular systolic dysfunction. There was no significant association among patients who has cardiac arrest, cardiogenic shock and arrhythmia. In this study mortality is significantly higher among the patients who has elevated haemoglobin A1c level. $(p=<.002)$

Previous studies have shown that an elevated $\mathrm{HbA1c}$ is associated with increased cardiovascular risk in patients with and without diabetes. Malmberg et al.(1999) found an association between elevated HbA1c and mortality after myocardial infarction, relative risk $(95 \% \mathrm{Cl}) 1.07$ $(1.01-1.21)^{8}$

In this study 93(82\%) patients were male and 34(18\%) were female. Female patients comprised a small part of the present study. Other studies also observed a small proportion of female patients. In Bangladesh, all most all of the studies reported an overwhelming majority of male patients. Asaduzzaman (2008) reported 22\%, Amanullah (1994) found $11.0 \%$ female in their study. The current study result correlates with these studies. 9,10

This study showed that the mean age of both HbA1c group was around $53 \pm 11$ years. Mokeddes (1997) reported similar age pattern of $54.0 \pm 9.9$ years and Rahim (1993) reported a mean age 52.5 years among the patients of STEMI. All these results were comparable with the present study.
STEMI Patients with elevated haemoglobin A1c level has $14 \%$ in hospital mortality in my study which is consistent with the findings of Haque, (2001) who showed around $12 \%$ in hospital mortality in the same STEMI population group. Haque, (2001) also showed that the main reason of death was pump failure and ventricular fibrillation. In my study, pump failure is also significantly higher in the study population who are in elevated haemoglobin A1c group. $^{2}$

Khaw et al. (2004) was carried out a study to examine the relationship between hemoglobin $\mathrm{A} 1 \mathrm{c}$, and mortality and found that, the relationship between hemoglobin A1c and short term mortality was continuous and significant. The relationship was apparent in persons without known diabetes. An increase in hemoglobin A1c of 1 percentage point was associated with a relative risk for death from any cause of $1.24(95 \% \mathrm{Cl}, 1.14$ to 1.34 ; P $<0.001)$ in men and with a relative risk of $1.28(\mathrm{Cl}, 1.06$ to 1.32; $\mathrm{P}<0.001$ ) in women. In my study, mortality was significantly higher in patients with elevated haemoglobin A1c level which is consistent with the above findings. ${ }^{11}$

Conclusion : An elevated $\mathrm{HbA1c}$, suggesting impaired glucose regulation, indicates a worse prognosis in patients with STEMI. Despite modern therapies for STEMI patient, elevated $\mathrm{HbA1c}$ conferred a significant excess adverse in hospital outcome risk following STEMI. This raises the question of whether better glycaemic control might improve outcome in such patients. STEMI patients who has haemoglobin HbA1c level d" 6.5 have better in hospital outcome compared to elevated (>6.5) haemoglobin A1c level.

\section{References :}

1. Lopez AD, Mathers CD, Ezzati M, Jamison DT, Murray CJ.Global and regional burden of disease and risk factors, 2001: systematic analysis of population health data.Lancet. 2006 May 27;367(9524):1747-57.

2. Haque SA. Detection of left ventricular diastolic dysfunction in first acute myocardial infarction by 
Doppler echocardiography. MD Thesis. Bangabandhu Sheikh Mujib Medical University (BSMMU) Dhaka; 2001.

3. Cakmak M, Cakmak N, Cetemen S, et al.The value of admission glycosylated hemoglobin level in patients with acute myocardial infarction.Can $\mathrm{J}$ Cardiol. 2008 May;24(5):375-8.

4. Lynch M, Gammage MD, Lamb P, Nattrass M, Pentecost BL.Acute myocardial infarction in diabetic patients in the thrombolytic era.Diabet Med. 1994 Mar;11(2):162-5.

5. Selvin E, Steffes MW, Zhu H, et al. Glycatedhemoglobin, diabetes, and cardiovascular risk in nondiabetic adults. N Engl J Med. 2010 Mar 4;362(9):800-11.

6. Zoghbi WA, Enriquez-Sarano M, Foster E, et al.; American Society of Echocardiography. Recommendations for evaluation of the severity of native valvular regurgitation with two-dimensional and Doppler echocardiography. J Am Soc Echocardiogr. 2003 Jul;16(7):777-802.

7. Use of Glycated Haemoglobin (HbA1c) in the Diagnosis of Diabetes Mellitus. Abbreviated Report of a WHO Consultation. Geneva: World Health Organization; 2011.

8. Malmberg K, Norhammar A, Wedel H, Rydén L.Glycometabolic state at admission: important risk marker of mortality in conventionally treated patients with diabetes mellitus and acute myocardial infarction: long-term results from the Diabetes and Insulin-Glucose Infusion in Acute Myocardial Infarction (DIGAMI) study.Circulation. 1999 May 25;99(20):2626-32.

9. Amanullah M. Intravenous thrombolytics in acute myocardial infarction. Bangladesh HeartJournal 1994;5(1):5-6.

10. Asaduzzaman K. Impaired fasting glucose and cardiogenic shock in patients with acutemyocardial infarction. MD Thesis. Sir Salimullah Medical College Dhaka; 2008.

11. Khaw KT, Wareham N, Bingham S, Luben R, Welch A, Day N.Association of hemoglobin A1c with cardiovascular disease and mortality in adults: the European prospective investigation into cancer in Norfolk. Ann Intern Med. 2004 Sep 21;141(6): 413-20. 